resource transformation, quality of products, environmental impact of livestock production and animal heath and welfare. Obviously, the classical approach to feeding animals "to meet their requirements and express their potential" is unable to tackle these new challenges. Therefore, this new situation needs a careful re-consideration of the classical feed unit systems (energy, protein...) and the ways to apply them.

\section{The concept of multiple responses to diets}

To take into consideration these new challenges, it is necessary to change our viewpoint in the sense that the target is now to add value to feed resources while optimizing the compromise between the above cited constraints. This new concept can be applied in practice if multiple responses (production, quality of products, feed efficiency, emissions on environment, animal health and welfare) of the animals to the diets or feeding practices are known and modelled. As a consequence, this new multicriteria evaluation system requires the following: 1 ) identifying the criteria that are to be optimized; 2 ) modelling the marginal responses of each criteria to the diets and dietary practices; 3) identifying the inherent relationships among the responses to each determinant, providing information on the possibilities of compromise between antagonistic responses. For example the fibre content of the diets of sows is now increasing to improve their well being status, however this choice decreases the dietary energy and thus the animal growth. Nitrogen concentration of feed is also an example that illustrates some antagonism that can exist between maximizing animal performance and reducing nitrogen emission via faeces and particularly urine; 4) providing the weights for criteria according to the importance accorded to them, even economical weights; 5) Defining tools to achieve these optimizations.

Consequences in building feed databases:

In turn, it is necessary to try to shift the concept of 'nutritive value' of feed (concentration in various key nutrients or feed units) to a new concept of 'response potential' of feed. For this, each feed would be characterized in terms of its contribution to response (i.e. environmental value....). It must be stressed that some classical components of nutritive value are also suited to predict responses. For instance OM digestibility is a key item to predict both nutritive (energy) value and feed efficiency. A major limit of characterizing the feed by the 'potential of responses' is that it assumes a basic principle of additivity. For instance, as global warming is becoming a major issue, classification of feeds based on their potential to produce methane could be a priori interesting. However, interactions between feed within diets are so marked that $\mathrm{CH} 4$ production cannot be a feed attribute. In contrast, it is a dietary characteristic.

New potential of responses of feed can be defined. For example, in warm areas, the ability of the feed to allow the animal to better fight against the heat would be an important criterion. This would indicate that it is necessary to differentiate between feeds on their ability to induce extra heat, on their capacity to buffer the water balance and minerals related to sweating.

The multicriteria approach should also incorporate the concept of risk to the animal, for instance under intensive conditions, ruminants face the risk of acidosis and some feed characteristics can allow reducing the risk of acidosis. Otherwise, risk for the whole food chain could also be considered for feed containing undesirable anti nutritive factors.

\section{Modeling multiple responses}

The multricriteria approach will take knowledge or information from systemic modelling. This allows us to integrate the endogenous and exogenous controls of metabolic flux which determine responses to the diet. Moreover systemic modelling presents animal responses under different context. The new concept of Feed multi criteria evaluation has to be included in the larger approach of farming system which is also evaluated for multiple functions.

\title{
Feed and diet value evaluation from literature data and from the existing databases
}

\author{
Daniel Sauvant ${ }^{1,2}$, Gilles Tran $^{2}$, Valérie Heuze ${ }^{2}$, Denis Bastianelli ${ }^{3}$ and Harry Archimede ${ }^{4}$ \\ ${ }^{1}$ AgroParisTech-INRA, UMR 791 MoSAR, 75231 Paris Cedex 05, France; ${ }^{2}$ Association Française de Zootechnie, 75231 Paris Cedex 05, France; ${ }^{3}$ CIRAD, Systèmes \\ d'élevage et produits animaux, 34398 Montpellier Cedex 05, France; ${ }^{4}$ INRA, UR143 Unité de Recherches Zootechniques, 97170 Petit-Bourg, France \\ Email: sauvant@agroparistech.fr
}

The production of information on feed characteristics is increasing rapidly and numerous data bases are now built in various organizations (research, universities, private companies...). From all these data, several methods can be applied to predict nutritive and feeding values of resources.
These methods are more or less easy to apply, accurate, repetable, costly, and there is a concern on the way to combine all this heterogenous information into a consistent frame to obtain ultimate reference values. The present feed tables, which were built through various ways, can differ 
largely for the referenced values of the same feed. The present communication focuses on some of the issues which appear in predicting feed values of tropical resources for ruminants. Other important aspects, such as chemical analysis, have been discussed in the plenary session.

Are in vitro and in vivo data equivalent? A first aspect adresses the level of equivalence between information from in vitro and in vivo data. In vivo digestibility of organic matter (OMD) is the key information to assess the feed nutritive value. However, it is fairly difficult and costly to carry out OMD, therefore in vitro methods are more and more frequently applied. From pooling data of the literature, it appears that in vitro and in vivo results are rather similar for feed having NDF content and OM digestibility in the ranges of $40-50 \% \mathrm{DM}$ and $55-65 \%$ respectively. For higher NDF contents, in vivo OMD values become higher than in vitro ones with differences that can be more than 20 points. This bias is probably the consequence of the adaptation of animals to rough feed (longer transit and chewing times, more $\mathrm{N}$ recycling...). Consequently, in vitro data must be used cautiously to predict in vivo OMD for feeds rich in the cell wall.

Usefulness of in sacco data? A second issue concerns the usefulness of values of in sacco, or in situ, degradation of feed constituents. This method has proved to be interesting to predict protein or starch digestion in the rumen and by-pass flows of the corresponding fractions of feed. Thus, a challenge is to pool the published in situ data to extract main values allowing the building of tables including reference values of in sacco effective degradability of $\mathrm{N}$, starch.... Moreover, since dietary indigestible NDF is the major determinant of OMD, the in sacco method can also be applied to predict NDF undigestibility. Comparison between in sacco and in vivo NDF undigestibility of rations is very encouraging. Thus in sacco can also be used to rank concentrate and by-product feed according to their (un)digestibility of NDF. This approach distinguishes feeds in 2 extreme groups according to their NDF undigestibility: more than $50 \%$ (cereal straws, hulls of rapeseed, peanut and sunflower...) and less than $20 \%$ (palm products, corn grain products, soybean hulls, citrus and beet pulp...). Only a few feed have an intermediary position between these two groups.

From feed to diet evaluation? A third aspect deals with the fact that tabulated nutritive values (NV) of feeds are assessed in standard conditions while, in practice, the target is to evaluate NV of diets. Diet NV is not the sum of the associated feed NV due to influences of various factors: feeding level (FL in terms of DMI\%LW), percentage of concentrate (\%CO), level of $\mathrm{N}$ supply to microbes in the rumen... Moreover for some items such as $\mathrm{CH} 4$, the influence of $\mathrm{FL}$ and \% $\% \mathrm{CO}$ are interacting and complicated, demonstrating that $\mathrm{CH} 4$ production cannot be a tabulated feed attribute. Thus, the prediction of dietary NV from feed is a complicated task requiring response functions to key factors of not only OMD or energy digestibility, but also energy flow like $\mathrm{CH} 4$ and urine. Estimation and standardization of these functions of response is an important issue for the future.

From nutritive to feeding values? Feeding value is generally assessed with the level of spontaneous DOM intake per $\mathrm{kgLW}^{0.75}$, thus it is approximatively the product of DMI $(\mathrm{kg} /$ $\mathrm{LW}^{0.75}$ ) and OMD which are also mutually linked. For forages, since the DMI values referenced in tables are measured into cages, an ultimate concern is the prediction of the actual value of DMI at pasture which could be somewhat different.

In conclusion, the contexts presented demonstrate that nutritive and feed value evaluation from the numerous available data has to be rigourously conducted and must be carefully traced to allow further improvements without having to re-start from zero.

\title{
Measurement methods for generating new data, the example of NIRS
}

\author{
Rob M Dixon ${ }^{1}$ and David B Coates ${ }^{2}$
}

\footnotetext{
${ }^{1}$ The University of Queensland, QAAFI, PO Box 6014, Rockhampton 4702, Australia; ${ }^{2}$ ATSIP, CSIRO Ecosystems Sciences, PMB PO Aitkenvale, Qld 4814, Australia Email : Rob.Dixon@deedi.qld.gov.au
}

Introduction Near infrared (NIR) spectroscopy is an analytical technique measuring light absorption in the $780-2500 \mathrm{~nm}$ region which is closely related to important chemical bonds $(\mathrm{OH}, \mathrm{NH}$ and $\mathrm{CH})$. NIR can be used to measure many nutritionally important constituents of concentrate and forage feedstuffs (Roberts et al. 2004; Andres et al. 2005).

Approach NIR spectroscopy depends on the development, in representative sets of samples, of mathematical relationships (calibration equations) between spectra and constituents or attributes of the samples measured by conventional chemistry. These calibrations are then applied to the spectra of unknown samples to estimate constituents or attributes of interest (Williams and Norris, 2001). NIR calibration equations tend to be specific to the circumstances of the data used for their development.

A major advantage of NIRS is that application allows rapid, routine and economical analysis of feedstuffs where appropriate calibrations are available for constituents of interest. Also, 